\title{
Alzheimer's Disease: Initial Clinical Implementation of Automated Volumetry
}

\author{
Diego A. Herrera, Julio Vargas, John Fredy Ochoa, Jon Edinson Duque, Sergio A. Vargas, \\ Francisco Lopera, Mauricio Castillo
}

Keywords: Alzheimer's disease, mild cognitive impairment, dementia, brain volumetry, magnetic resonance imaging

doi:10.1017/cjn.2014.23

Can J Neurol Sci. 2014; 41: 651-653

\section{INTRODUCTION}

Alzheimer's disease (AD) is a chronic, progressive, and degenerative brain disease. Mild cognitive impairment (MCI) may represent a transitional predementia state between normal aging and $\mathrm{AD}$ dementia. Noninvasive diagnostic methods are necessary to identify asymptomatic individuals and MCI subjects with high-risk genetic factors who are candidates for early preventive or therapeutic intervention.

Structural MRI methods allow visualization of macroscopic cerebral atrophy secondary to AD cellular changes. To be a diagnostic biomarker, MRI should be able to detect and quantify essential characteristics in high-risk patients (e.g., amnestic MCI individuals or asymptomatic carriers of a high-risk genetic factor with familial AD history) and in patients with clinical AD diagnosis. It is also important to accurately differentiate between healthy control (HC) subjects and AD dementia and MCI individuals. Manually defined MRI regions of interest or automated methods can be used to identify AD and MCI individuals. ${ }^{1}$ Image analysis algorithms have allowed the development of MRI-based tools to quantify atrophy in various anatomical regions by automatic segmentation. ${ }^{2}$

In 2009, Desikan and colleagues ${ }^{3}$ described the use of an automated volumetry tool for AD diagnosis with excellent clinical and neuropsychological correlation (specificity, 91\%; sensitivity, $90 \%$ ). Our goal was to explore the possible implementation of this tool and its usefulness in patients with clinical and neuropsychological diagnosis of MCI and AD dementia.

\section{Materials ANd Methods}

\section{Clinical Evaluation}

Clinical neurological examination was performed by a neurologist with more than 20 years of experience in a dementia clinic (FL). The CERAD (Consortium to Establish a Registry for Alzheimer's Disease) clinical and neuropsychology assessment battery was applied.

\section{Image Acquisition}

Institution review board approval and signed informed consent were obtained. Magnetic resonance images were acquired with a 1.5-T scanner (Avanto, Siemens, Erlangen, Germany). A wholebrain Sagittal T1 MPRAGE sequence $(\mathrm{FOV}=240$, matrix $=192 \times$ 192, resolution $=1.3 \mathrm{~mm}^{3}, \mathrm{TR}=1670 \mathrm{~ms}, \mathrm{TE}=3.6 \mathrm{~ms}$, flip angle $=8^{\circ}, \quad \mathrm{TI}=1000 \mathrm{~ms}, \quad$ averages $=2,128$ slices, scanning time $=4 \mathrm{~min}, 39 \mathrm{~s}$ ) was acquired in patients with $\mathrm{AD}$ dementia, MCI, and presymptomatic carriers of the $\mathrm{E} 280 \rightarrow$ A presenilin-1 mutation.

\section{Automated Volumetry}

FreeSurfer software is an automated tool set for cortical surface reconstruction of structural MRI data developed at the Athinoula A. Martinos Center for Biomedical Imaging (Harvard-Massachusetts Institute of Technology, Division of Health Sciences and Technology). With a 16-GB RAM 8-processor Linux server, the automatic reconstruction scheme for single-subject analysis was performed in two patients (one with AD dementia and one with MCI). Entorhinal, hippocampal, and supramarginal cortical area, as well as thickness and volume, were calculated. Processing time was $26 \mathrm{~h}$ (both subjects were simultaneously processed). Postprocessing was reviewed by a neuroradiologist (DAH) to verify segmentation accuracy and appropriate cortex labeling.

\section{Volume Correction}

Hippocampal volume was corrected according to intracranial volume (eTIV) to remove skull size effect on parcelated volumes. The following formula was used:

corrected hippocampal volume $=$ (total hippocampal volume $/$ eTIV) $\times 1000$

\section{Analysis}

Obtained values were compared with established ranges for $\mathrm{AD}, \mathrm{MCI}$, and HCs. Reference values were as follows: entorhinal cortex thickness for $\mathrm{AD}=4.97$ (standard deviation [SD], 0.68), $\mathrm{MCI}=5.80(\mathrm{SD}, 0.78), \mathrm{HC}=6.96(\mathrm{SD}, 0.44)$; supramarginal gyrus thickness for $\mathrm{AD}=4.26(\mathrm{SD}, 0.33), \mathrm{MCI}=4.30(\mathrm{SD}, 0.34)$, $\mathrm{HC}=4.68(\mathrm{SD}, 0.34)$; and hippocampal volume for $\mathrm{AD}=3.35$ $(\mathrm{SD}, 0.59), \mathrm{MCI}=3.68(\mathrm{SD}, 0.63), \mathrm{HC}=4.76(\mathrm{SD}, 0.65){ }^{3}$

From the Department of Radiology (DAH, SAV), Universidad de Antioquia, Medellín Colombia; Grupo de Bioelectrónica e Ingeniería Clínica (JFO, JED), Universidad de Antioquia, Medellín, Colombia; Department of Radiology (JED), CediMed (Centro Avanzado de Diagnóstico Médico), Medellín, Colombia; Department of Radiology (JV), Universidad del Valle, Cali, Colombia; Grupo de Neurociencias de Antioquia (FL), Medellín, Colombia; Department of Radiology (MC), University of North Carolina, Chapel Hill, North Carolina, USA.

Received March 10, 2014. Final Revisions Submitted April 29, 2014. Correspondence to: Diego A. Herrera, Department of Radiology, Universidad de Antioquia, Medellín, Colombia. E-mail: herrera.diego@gmail.com 


\section{RESULTS}

\section{Case 1}

A 47-year-old male patient presented with 5 years of cognitive impairment and clinical diagnosis of AD dementia. The patient was carrier of the E280 $\rightarrow$ A presenilin-1 mutation. MRI showed global and hippocampal atrophy (Figure 1). Fazekas 1 (punctate nonconfluent lesions) white matter changes were present. Volumetry values for hippocampal volume, entorhinal cortex thickness, and supramarginal cortex thickness (Table 1) were within the AD range.

\section{Case 2}

A 46-year-old female patient presented with 2 years of memory loss. Clinical and neuropsychological assessment was indicative of MCI. MRI (Figure 1) showed mild hippocampal atrophy (in a lower degree than the individual with AD dementia). Mild white matter changes (Fazekas 1) were noted. Volumetric values for hippocampal volume, entorhinal cortex thickness, and supramarginal cortex thickness (Table 1) were within the MCI range.

When comparing the various measurements of MCI and AD subjects with $\mathrm{HCs}$, we noted a progressive decrease in the three variables (hippocampal volume, entorhinal cortex thickness, and supramarginal cortex thickness) as cognitive deficit increased (Figure 2).

\section{DiscuSSION}

The neuroimaging marker for $\mathrm{AD}$ is cortical atrophy secondary to neuronal degeneration. Brain volume loss is diffuse, bilateral, and symmetrical, most prominent in parietal and temporal lobes involving the hippocampus. White matter volume is reduced by Wallerian degeneration. Regarding atrophy spatiotemporal distribution, entorhinal areas with involvement of the hippocampus are compromised first, and then volume loss progresses from the limbic system to temporal and parietal association cortex and eventually the entire neocortex can be involved. ${ }^{4}$

Mild cognitive impairment may represent a transitional state between normal aging and $\mathrm{AD}$ dementia. Up to $80 \%$ of $\mathrm{MCI}$ amnestic-subtype patients progress to dementia after 6 years, requiring noninvasive in vivo diagnostic methods to identify MCI individuals who can be candidates for prompt therapeutic interventions. ${ }^{3}$ A recent study showed the capability of clinical evaluation combined with MRI volumetric measurements to predict conversion from MCI to AD dementia. ${ }^{5}$

In clinical practice, a fast, economical, and accurate method for volumetric measurements of the hippocampus is essential. However, hippocampal complex shape and ambiguous boundaries complicate manual measurement, which requires time and experience. An alternative method is the automated segmentation process. Software applications-such as FreeSurfer, Individual Brain Atlases using Statistical Parametric Mapping (IBASPM), and Oxford Centre for Functional MRI of the Brain (FMRIB) software library (FSL) - provide automatic hippocampus segmentation tools, which some studies have validated. ${ }^{5}$ In two studies, FreeSurfer slightly overestimated hippocampal volume in healthy and depressed subjects; however, correlation was better than with FSL and IBASPM. ${ }^{5}$

FreeSurfer segmentation tool has demonstrated robust performance in relation to the anatomical variability even in $\mathrm{AD}$ patients. A FreeSurfer software validation study showed good correlation between the automated and manual measurements. ${ }^{5}$ This result is related to the absence of human intervention and process repeatability with clear advantages in the automated process. However, FreeSurfer has limitations in evaluating certain
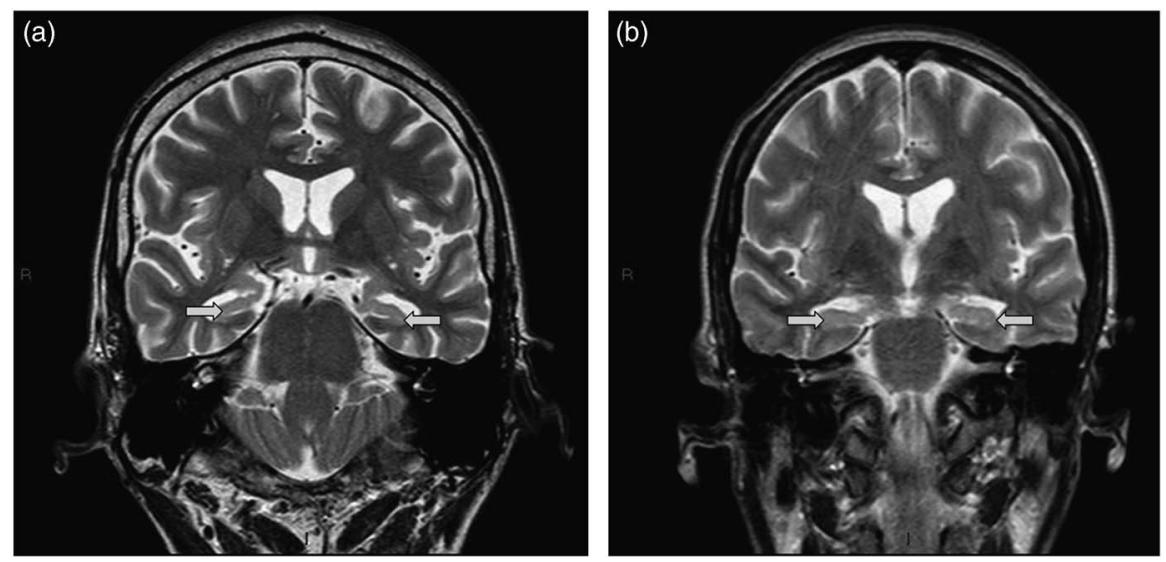

Figure 1: T2-weighted coronal images show hippocampal atrophy (arrows) with greater compromise in $A D$ patient $(A)$ than in MCI subject $(B)$.

Table 1: Brain volumetry

\begin{tabular}{|c|c|c|c|}
\hline Subject(s) & Entorhinal cortex thickness & Supramarginal gyrus thickness & Hippocampal vol \\
\hline Case 1 (AD Dementia) & 5.06 & 4.37 & 3.03 \\
\hline Case 2 (MCI) & 5.72 & 4.64 & 3.84 \\
\hline Healthy control subjects (reference values) & 6.96 & 4.68 & 4.76 \\
\hline
\end{tabular}



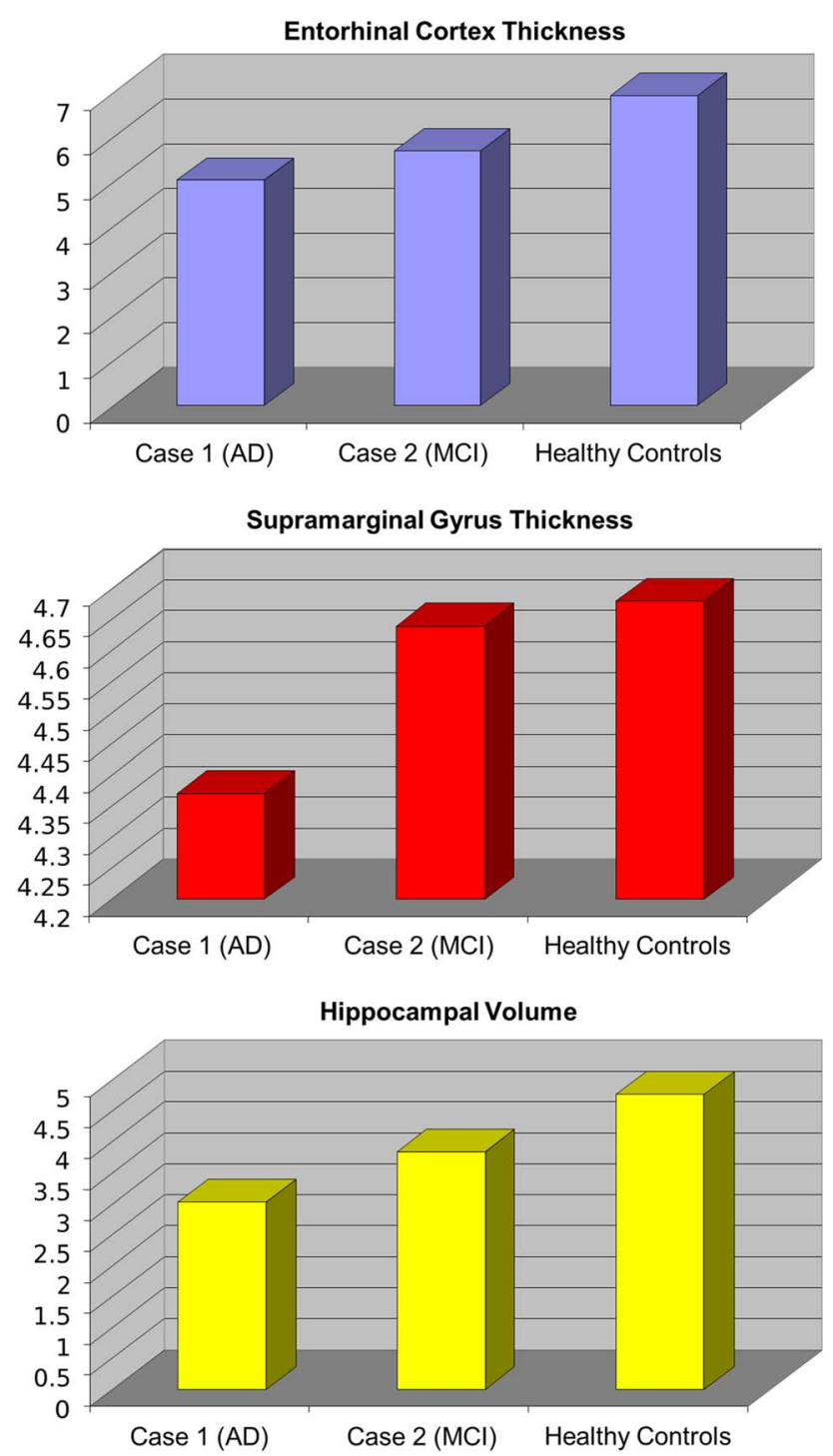

Figure 2: Volumetric measurements between $\mathrm{HCs}$, MCI subject, and $A D$ dementia patient.

mesial temporal regions such as the amygdala, where the segmentation has been more equivocal than the hippocampus. ${ }^{6}$ This finding could be a significant drawback of the method because early AD can affect the amygdala. ${ }^{7}$

As Desikan and colleagues ${ }^{3}$ did in 2009, we observed good correlation in our cases between automated measurements by FreeSurfer and clinical and neuropsychological tests for diagnosis of MCI and AD dementia. These findings suggest the feasibility of using MRI-based software tools as a diagnostic marker of AD in individual cases. High consistency and reproducibility of the method suggest it could be implemented in clinical practice to diagnose those pathologies.

A limitation of this study is that it did not quantitatively evaluate white matter changes and other variables such as microhemorrhage that frequently are present in the MRI of patients with cognitive deficits. Microvascular disease commonly coexists with $\mathrm{AD}$, and mixed dementia is prevalent. Future studies may include hippocampal automated volumetry together with segmentation and quantification of white matter lesions to establish correlation between these variables and the neuropsychological profile of those subjects. However, because significant vascular changes are rare in early-onset genetic forms of $\mathrm{AD}$ - as in later-onset sporadic $\mathrm{AD}$ or mixed dementia - this limitation is somewhat mitigated.

\section{ConCLusion}

In individual cases, the described technique can be implemented in clinical practice to diagnose $\mathrm{AD}$ dementia and MCI as a complement to neuropsychological test. The most useful variables for differentiation between the two conditions were hippocampal volume and entorhinal cortex thickness. Also, these parameters may be useful for patient follow-up and to determine atrophy progression.

\section{Disclosures}

The authors certify that no actual or potential conflict of interest exists in relation to this article. This material has not been presented at any meeting.

The authors declare no grant support (including NIH funding).

\section{REFERENCES}

1. Devanand DP, Pradhaban G, Liu X, et al. Hippocampal and entorhinal atrophy in mild cognitive impairment: prediction of Alzheimer disease. Neurology. 2007;68:828-36.

2. Fischl B, Salat DH, Busa E, et al. Whole brain segmentation: automated labeling of neuroanatomical structures in the human brain. Neuron. 2002;33:341-55.

3. Desikan RS, Cabral HJ, Hess CP, et al; Alzheimer's Disease Neuroimaging Initiative. Automated MRI measures identify individuals with mild cognitive impairment and Alzheimer's disease. Brain. 2009;132:2048-57.

4. Vernooij MW, Smits M. Structural neuroimaging in aging and Alzheimer's disease. Neuroimag Clin N Am. 2012;22:33-55.

5. Sánchez-Benavides G, Gómez-Ansón B, Sainz A, Vives Y, Delfino M, Peña-Casanova J. Manual validation of FreeSurfer's automated hippocampal segmentation in normal aging, mild cognitive impairment, and Alzheimer disease subjects. Psychiatry Res. 2010;181:219-25.

6. Morey RA, Petty CM, Xu Y, et al. A comparison of automated segmentation and manual tracing for quantifying hippocampal and amygdala volumes. Neuroimage. 2009;45:855-66.

7. Klein-Koerkamp Y, Heckemann RA, Ramdeen KT, et al. Amygdalar atrophy in early Alzheimer's disease. Curr Alzheimer Res. 2014;11:239-52. 